\title{
ON THE WEIGHTED FORWARD REDUCED VOLUME OF RICCI FLOW
}

\author{
LIANG CHENG AND ANQIANG ZHU
}

(Communicated by Lei $\mathrm{Ni}$ )

\begin{abstract}
In this paper, we introduce the weighted forward reduced volume of Ricci flow. The weighted forward reduced volume, which is related to expanders of Ricci flow, is well-defined on noncompact manifolds and monotone nonincreasing under Ricci flow. Moreover, we show that, just the same as Perelman's reduced volume, the weighted reduced volume entropy has the value $(4 \pi)^{\frac{n}{2}}$ if and only if the Ricci flow is the trivial flow on flat Euclidean space.
\end{abstract}

\section{INTRODUCTION}

In [11, G. Perelman introduced the reduced entropy (i.e. reduced distance and reduced volume), which becomes one of the powerful tools for studying Ricci flow. The reduced entropy enjoys very nice analytic and geometric properties, including in particular the monotonicity of the reduced volume. These properties can be used, as demonstrated by Perelman, to show that the limit of the suitable rescaled Ricci flows is a gradient shrinking soliton.

Then M. Feldman, T. Ilmanen, and L. Ni [3] made an interesting observation that there is a dual version of Perelman's reduced entropy which is related to the expanders of Ricci flow. Let $g(t)$ solve the Ricci flow

$$
\frac{\partial g}{\partial t}=-2 R c
$$

on $M \times[0, T]$. Fix $x \in M^{n}$ and let $\gamma$ be a path $(x(\eta), \eta)$ joining $(x, 0)$ and $(y, t)$. They define the forward $\mathcal{L}_{+}$-length as

$$
\mathcal{L}_{+}(\gamma)=\int_{0}^{t} \sqrt{\eta}\left(R(\gamma(\eta))+\left|\gamma^{\prime}(\eta)\right|^{2}\right) d \eta
$$

Denote $L_{+}(y, t)$ to be the length of a shortest forward $\mathcal{L}_{+}$-length joining $(x, 0)$ and $(y, t)$. Let

$$
l_{+}(y, t)=\frac{L_{+}(y, t)}{2 \sqrt{t}}
$$

Received by the editors October 31, 2011.

2010 Mathematics Subject Classification. Primary 53C44.

Key words and phrases. Ricci flow, weighted forward reduced volume, Type III singularities, gradient expanding soliton.

The first author was supported by NSF grant of China No. 11171126 and self-determined research funds of CCNU from the colleges basic research and operation of MOE CCNU11A01027.

The second author was supported by NSF grant of China No. 11126190. 
be the forward $l_{+}$-length. Note that the forward reduced distance (1.3) is defined under the forward Ricci flow (1.1), which is the only difference from Perelman's reduced distance defined under the backward Ricci flow. The forward reduced volume is defined in [3] as

$$
\theta_{+}(t)=\int_{M}(t)^{-\frac{n}{2}} e^{l_{+}(y, t)} d \operatorname{vol}(y) .
$$

They also proved that the forward reduced volume defined in (1.4) is monotone nonincreasing along the Ricci flow (1.1).

Note that the forward reduced volume defined in (1.4) may not be well-defined on noncompact manifolds (see [3]). In this paper, we introduce the weighted forward reduced volume based on the work in [3] and [11. We shall prove that the weighted forward reduced volume, which is related to the expanders of the Ricci flow, is welldefined on noncompact manifolds and monotone nonincreasing under the Ricci flow (1.1).

We define the weighted forward reduced volume as follows. First, we define the forward $\mathcal{L}_{+}$-exponential map $\mathcal{L}_{+} \exp (V, t): T_{x} M \rightarrow M$ at time $t \in[0, T)$ as follows. For $V \in T_{x} M$, let $\gamma_{V}$ denote the $\mathcal{L}_{+}$-geodesic such that $\gamma_{V}(0)=p$, $\lim _{t \rightarrow 0} \sqrt{t} \gamma_{V}^{\prime}(t)=V$. If $\gamma_{V}$ exists on $[0, t]$, we set

$$
\mathcal{L}_{+} \exp (V, t)=\gamma_{V}(t) .
$$

Denote $\tau_{V}$ to be the first time the $\mathcal{L}_{+}$-geodesic $\gamma_{V}$ stops minimizing. Define

$$
\Omega(t)=\left\{V \in T_{x} M^{n}: \tau_{V}>t\right\} .
$$

Obviously, $\Omega\left(t_{2}\right) \subset \Omega\left(t_{1}\right)$ if $t_{1}<t_{2}$. Let $J_{i}^{V}(t), i=1, \cdots, n$, be $\mathcal{L}_{+}$-Jacobi fields along $\gamma_{V}(t)$ with

$$
J_{i}^{V}(0)=0,\left(\nabla_{V} J_{i}^{V}\right)(0)=E_{i}^{0},
$$

where $\left\{E_{i}^{0}\right\}_{i=1}^{n}$ is an orthonormal basis for $T_{x} M$ with respect to $g(0)$. Then $D\left(\mathcal{L}_{+} \exp (V, t)\right)\left(E_{i}^{0}\right)=J_{i}^{V}(t)$. We define

$$
\mathcal{L}_{+} J_{V}(t)=\sqrt{\operatorname{det}\left(\left\langle J_{i}^{V}(t), J_{j}^{V}(t)\right\rangle\right)}
$$

and the weighted forward reduced volume as

$$
\widetilde{\mathcal{V}}_{+}(t)=\int_{\Omega(t)} t^{-\frac{n}{2}} e^{l_{+}\left(\gamma_{V}(t), t\right)} e^{-2|V|_{g(0)}^{2}} \mathcal{L}_{+} J_{V}(t) d x_{g(0)}(V)
$$

where $d x_{g(0)}$ is the standard Euclidean volume form on $\left(T_{x} M, g(x, 0)\right)$; i.e. we define the weighted forward reduced volume as

$$
\widetilde{\mathcal{V}}_{+}(t)=\int_{M} t^{-\frac{n}{2}} e^{l_{+}(y, t)} e^{-2\left|\mathcal{L}_{+} \exp ^{-1}(y, t)\right|_{g(0)}^{2}} d v o l(y),
$$

where we set $V=\mathcal{L}_{+} \exp ^{-1}(y, t)$. We use the convention

$$
\mathcal{L}_{+} J_{V}(t) \doteq 0 \text { for } t \geq \tau_{V} .
$$

Then we can write the weighted forward reduced volume as

$$
\widetilde{\mathcal{V}}_{+}(t)=\int_{T_{x} M^{n}} t^{-\frac{n}{2}} e^{l_{+}\left(\gamma_{V}(t), t\right)} \mathcal{L}_{+} J_{V}(t) e^{-2|V|_{g(0)}^{2}} d x_{g(0)}(V) .
$$

We remark that the density of the forward reduced volume (1.4) (i.e. $\left.(t)^{-\frac{n}{2}} e^{l_{+}(y, t)} \operatorname{dvol}(y)\right)$ is not pointwise monotone nonincreasing under the Ricci flow 
(1.1). So it is not easy for us to add the weighted term to the forward reduced volume such that it could be defined on noncompact manifolds. In order to overcome this problem, we employ Perelman's technique [11] where we pull the density of the forward reduced volume back to the tangent space by the $\mathcal{L}_{+}$-exponential map (here we define the $\mathcal{L}_{+}$-exponential map in a similar way to [11]). Then we prove that the forward reduced volume density

$$
d \mathcal{V}_{+}=t^{-\frac{n}{2}} e^{l_{+}\left(\gamma_{V}(t), t\right)} \mathcal{L}_{+} J_{V}(t) d x_{g(0)}(V)
$$

is pointwise monotone nonincreasing under the Ricci flow (1.1) with respect to $V \in T_{x} M$ (see Theorem 2.3). Notice that (see the proof of Theorem 1.1)

$$
\lim _{t \rightarrow 0^{+}} t^{-\frac{n}{2}} e^{l_{+}\left(\gamma_{V}(t), t\right)} \mathcal{L}_{+} J_{V}(t)=2^{n} e^{|V|_{g(0)}^{2}}
$$

So we only need to add the weighted term $e^{-2|V|_{g(0)}^{2}}$ to the density of the forward reduced volume, which guarantees that the weighted forward reduced volume we defined in (1.8) is well-defined on noncompact manifolds at $t=0$. Moreover, the weighted forward reduced volume is monotone nonincreasing under the Ricci flow (1.1) since we have

$$
\frac{d}{d t}\left(t^{-\frac{n}{2}} e^{l_{+}\left(\gamma_{V}(t), t\right)} \mathcal{L}_{+} J_{V}(t) e^{\left.-2|V|_{g(0)}^{2}\right)}=e^{-2|V|_{g(0)}^{2}} \frac{d}{d t}\left(t^{-\frac{n}{2}} e^{l_{+}\left(\gamma_{V}(t), t\right)} \mathcal{L}_{+} J_{V}(t)\right) \leq 0\right.
$$

for $V \in \Omega(t)$.

We have the following exact properties for the weighted forward reduced volume.

Theorem 1.1. The weighted forward reduced volume defined in (1.8) is monotone non-increasing under the Ricci flow (1.1) and $\widetilde{\mathcal{V}}_{+}(t) \leq \lim _{t \rightarrow 0+} \widetilde{\mathcal{V}}_{+}(t) \leq(4 \pi)^{\frac{n}{2}}$. If $\tilde{\mathcal{V}}_{+}\left(t_{1}\right)=\widetilde{\mathcal{V}}_{+}\left(t_{2}\right)$ for some $0<t_{1}<t_{2}$, then this flow is a gradient expanding soliton on $0 \leq t<\infty$ and hence is the trivial flow on flat Euclidean space. In particular, if $\widehat{\widetilde{\mathcal{V}}}_{+}(\bar{t})=(4 \pi)^{\frac{n}{2}}$ for some time $\bar{t}>0$, then this flow is the trivial flow on flat Euclidean space.

We also have the following rescaling property for the weighted forward reduced volume.

Theorem 1.2. We have $\widetilde{\mathcal{V}}_{+}^{j}(t)=\widetilde{\mathcal{V}}_{+}\left(\lambda_{j}^{-1} t\right)$ under the rescaling $g_{j}(t)=\lambda_{j} g\left(\lambda_{j}^{-1} t\right)$, where $\widetilde{\mathcal{V}}_{+}^{j}$ and $\widetilde{\mathcal{V}}_{+}$denote the weighted forward reduced volume with respect to the metrics $g_{j}$ and $g$ respectively.

The organization of the paper is as follows. In section 2, we first recall some basic formulas and properties about forward reduced entropy in 3 . Then we study the properties of forward reduced volume density which are defined by the forward $\mathcal{L}_{+}$-exponential map. Finally, we give the proofs of Theorem 1.1 and Theorem 1.2. In the appendix of this paper, we give the proof of Theorem 2.1 and Theorem 2.2 , which are proved in [3], for the sake of completeness.

\section{WEIGHTED FORWARD REDUCED VOLUME AND EXPANDERS}

Before we present the proofs of Theorem 1.1 and Theorem 1.2 we recall some basic formulas and properties about forward reduced entropy found in [3]. Clearly, one can show that the $l_{+}$-length $l_{+}(y, t)$ is a locally Lipschitz function and the cut locus of $\mathcal{L}_{+} \exp (V, t)$ is a closed set of measure zero by using methods similar to those in 14 . 
We need the following two theorems due to Feldman, Ilmanen, and Ni [3], which state the following adapted form. We will give the proof of Theorem 2.1 and Theorem 2.2 in the appendix of this paper for the sake of completeness.

Theorem 2.1 ([3]). Let $\gamma$ be a path $(x(\eta), \eta)$ joining $(x, 0)$ and $(y, t)$. Denote $L_{+} \doteq L_{+}(y, t)$ to be the forward $\mathcal{L}_{+}$-length joining $(x, 0)$ and $(y, t)$. Set $X=\gamma^{\prime}(t)$ and let $Y$ be a variational vector along $\gamma$ such that $Y(0)=0$. The first variation of $\mathcal{L}_{+}$is that

$$
\delta \mathcal{L}_{+}=2 \sqrt{t}\langle X, Y\rangle(t)+\int_{0}^{t} \sqrt{\eta}\left\langle Y, \nabla R-2 \nabla_{X} X+4 R c(X, \cdot)-\frac{1}{\eta} X\right\rangle d \eta .
$$

If $\gamma(t)$ is the mimimal $\mathcal{L}_{+-}$geodesic, then

$$
\begin{gathered}
\nabla L_{+}=2 \sqrt{t} X, \\
t^{\frac{3}{2}}\left(R+|X|^{2}\right)=K+\frac{1}{2} L_{+},
\end{gathered}
$$

where $K=\int_{0}^{t} \eta^{\frac{3}{2}} H(X) d \eta$, and $H(X)=\frac{\partial R}{\partial t}+2\langle\nabla R, X\rangle+2 R c(X, X)+\frac{R}{t}$. The second variation of $\mathcal{L}_{+}$is that

$$
\begin{aligned}
\delta_{Y}^{2} \mathcal{L}_{+}= & 2 \sqrt{t}\langle X, Y\rangle(t)+\int_{0}^{t} \sqrt{\eta}(\operatorname{HessR}(Y, Y)-2 R(X, Y, X, Y) \\
& \left.+2\left|\nabla_{X} Y\right|^{2}+4 \nabla_{Y} R c(Y, X)-2 \nabla_{X} R c(Y, Y)\right) d \eta
\end{aligned}
$$

Let $\tilde{Y}$ be a vector field along $\gamma$ that satisfies the $O D E$

$$
\left\{\begin{array}{l}
\nabla_{X} \tilde{Y}(\eta)=R c(\tilde{Y}(\eta), \cdot)+\frac{1}{2 \eta} \tilde{Y}(\eta), \eta \in[0, t] \\
\tilde{Y}(0)=Y(0)=0
\end{array}\right.
$$

Then

$$
H \operatorname{Hess} L_{+}(\tilde{Y}, \tilde{Y}) \leq \frac{|\widetilde{Y}|^{2}}{\sqrt{t}}+2 \sqrt{t} R c(\tilde{Y}, \tilde{Y})-\int_{0}^{t} \sqrt{\eta} H(X, \tilde{Y}) d \eta
$$

where $H(X, \tilde{Y})=-\operatorname{HessR}(\tilde{Y}, \tilde{Y})+2 R(X, \tilde{Y}, X, \tilde{Y})+2|R c(X, \tilde{Y})|^{2}+\frac{R c(\widetilde{Y}, \widetilde{Y})}{t}+$ $2 \frac{\partial R c}{\partial t}(\tilde{Y}, \tilde{Y})-4 \nabla_{\tilde{Y}} R c(\tilde{Y}, X)+4 \nabla_{X} R c(\tilde{Y}, \tilde{Y})$. The equality holds in (2.6) if and only if the vector field $\widetilde{Y}$ satisfying (2.5) is an $\mathcal{L}_{+}$-Jacobi field.

Theorem $2.2(\underline{3]})$. Let $\left.l_{+} \doteq l_{+}(y, t)\right)=\frac{L_{+}(y, t)}{2 \sqrt{t}}$ be the $l_{+}$-length from $(x, 0)$ to $(y, t)$. If $(y, t)$ is not in the cut locus of $\mathcal{L}_{+}$exp, then at $(y, t)$

$$
\begin{array}{r}
\frac{\partial l_{+}}{\partial t}=R-\frac{l_{+}}{t}-\frac{K}{2 t^{\frac{3}{2}}}, \\
\left|\nabla l_{+}\right|^{2}=\frac{l_{+}}{t}-R+\frac{K}{t^{\frac{3}{2}}}, \\
\Delta l_{+} \leq R+\frac{n}{2 t}-\frac{K}{2 t^{\frac{3}{2}}}, \\
\frac{\partial l_{+}}{\partial t}+\Delta l_{+}+\left|\nabla l_{+}\right|^{2}-R-\frac{n}{2 t} \leq 0, \\
2 \Delta l_{+}+\left|\nabla l_{+}\right|^{2}-R-\frac{l_{+}+n}{t} \leq 0 .
\end{array}
$$


We first study properties of the forward reduced volume density defined as

$$
d \mathcal{V}_{+}=t^{-\frac{n}{2}} e^{l_{+}\left(\gamma_{V}(t), t\right)} \mathcal{L}_{+} J_{V}(t) d x_{g(0)}(V),
$$

where $\mathcal{L}_{+} J_{V}(t)$ is defined in (1.7).

Note that the weighted forward reduced volume is

$$
\widetilde{\mathcal{V}}_{+}(t)=\int_{T_{x} M^{n}} e^{-2|V|_{g(0)}^{2}} d \mathcal{V}_{+}
$$

Analogously to [11, we have the following theorem.

Theorem 2.3. The forward reduced volume density $d \mathcal{V}_{+}$defined in (2.12) is monotone nonincreasing along the Ricci flow (1.1). Moreover, if $d \mathcal{V}_{+}\left(t_{1}\right)=d \mathcal{V}_{+}\left(t_{2}\right)$ for some $0<t_{1}<t_{2}$, then this flow is a gradient expanding soliton.

Proof. Let $\gamma_{V}(t)$ be the minimal $\mathcal{L}_{+}$-geodesic defined in (1.5) and $y=\gamma_{V}(t)$. We consider $(y, t)$ in the cut locus of $\mathcal{L}_{+} \exp (V, t)$. Recall that $\nabla l_{+}(y, t)=\gamma_{V}^{\prime}(t)=X(t)$. Then by (2.3) and (2.7), we get

$$
\begin{aligned}
\frac{d l_{+}\left(\gamma_{V}(t), t\right)}{d t} & =\frac{\partial l_{+}(y, t)}{\partial t}+\nabla l \cdot X \\
& =R-\frac{l_{+}(y, t)}{t}-\frac{K}{2 t^{\frac{3}{2}}}+|X|^{2} \\
& =\frac{1}{2} t^{-\frac{3}{2}} K .
\end{aligned}
$$

For any fixed $t$, we choose an orthonormal basis $\left\{E_{i}(t)\right\}$ of $T_{\gamma_{V}(t)} M$. We extend $E_{i}(\eta), \eta \in[0, t]$, to an $\mathcal{L}_{+}$-Jacobi field along $\gamma_{V}$ with $E_{i}(0)=0$. We write $J_{i}^{V}(t)=$ $\sum_{i}^{n} A_{i}^{j} E_{j}(t)$ for some matrix $\left(A_{i}^{j}\right) \in G L(n, \mathbb{R})$. Then $J_{i}^{V}(\eta)=\sum_{i}^{n} A_{i}^{j} E_{j}(\eta)$ for all $\eta \in[0, t]$.

Hence, by (2.6), we calculate at time $t$ :

$$
\begin{aligned}
\left.\frac{d}{d \eta}\right|_{\eta=t} \ln \mathcal{L}_{+} J_{V} & =\left.\frac{d}{d \eta}\right|_{\eta=t} \ln \sqrt{\operatorname{det}\left(\left\langle\sum_{k=1}^{n} A_{i}^{k} E_{k}, \sum_{l=1}^{n} A_{j}^{l} E_{l}\right\rangle\right)} \\
& =\left.\frac{1}{2} \frac{d}{d \eta}\right|_{\eta=t} \sum_{i}\left|E_{i}\right|^{2} \\
& =\sum_{i}\left(-\operatorname{Rc}\left(E_{i}, E_{i}\right)+\left\langle\nabla_{E_{i}} X, E_{i}\right\rangle\right) \\
& =\sum_{i}\left(-\operatorname{Rc}\left(E_{i}, E_{i}\right)+\frac{1}{2 \sqrt{t}} \operatorname{Hess} L_{+}\left(E_{i}, E_{i}\right)\right) \\
& \leq \sum_{i}\left(\frac{1}{2 t}-\frac{1}{2 \sqrt{t}} \int_{0}^{t} \sqrt{\eta} H\left(X, \widetilde{E}_{i}\right) d \eta\right),
\end{aligned}
$$

where $\widetilde{E}_{i}(\eta)$ are the vector fields along $\gamma_{V}$ satisfying

$$
\left\{\begin{array}{l}
\nabla_{X} \widetilde{E}_{i}(\eta)=R c\left(\widetilde{E}_{i}(\eta), \cdot\right)+\frac{1}{2 \eta} \widetilde{E}_{i}(\eta), \eta \in[0, t], \\
\widetilde{E}_{i}(t)=E_{i}(t)
\end{array}\right.
$$


which in particular implies that

$$
\left\langle\widetilde{E}_{i}, \widetilde{E}_{j}\right\rangle(\eta)=\frac{\eta}{t}\left\langle E_{i}, E_{j}\right\rangle(t)=\frac{\eta}{t} \delta_{i j} .
$$

It follows that

$$
H\left(X, \widetilde{E}_{i}\right)(\eta)=\frac{\eta}{t} H(x) .
$$

Hence

$$
\left.\frac{d}{d \eta}\right|_{\eta=t} \ln \mathcal{L}_{+} J_{V} \leq \frac{n}{2 t}-\frac{1}{2} t^{-\frac{3}{2}} K
$$

and

$$
\frac{d}{d t} \ln d \mathcal{V}_{+}=-\frac{n}{2 t}+\frac{\partial l+}{\partial t}+\frac{d \ln \mathcal{L}_{+} J_{V}}{d t} \leq 0 .
$$

If equality in (2.18) holds, then we have that equality in (2.15) holds. By Theorem 2.1. we conclude that each $\widetilde{E}_{i}(\eta)$ is an $\mathcal{L}_{+}$-Jacobi field. Hence

$$
\left.\frac{d}{d \eta}\right|_{\eta=t}\left|E_{i}\right|^{2}=\left.\frac{d}{d \eta}\right|_{\eta=t}\left|\widetilde{E}_{i}\right|^{2}=\frac{\left|E_{i}(t)\right|^{2}}{t} .
$$

Combining this with (2.14) and (2.19), we get

$$
R c\left(E_{i}, E_{i}\right)-\frac{1}{2 \sqrt{t}} \operatorname{HessL}_{+}\left(E_{i}, E_{i}\right)=-\frac{\left|E_{i}\right|^{2}}{2 t} .
$$

Now we can give the proof of Theorem 1.1

Proof of Theorem 1.1. By Theorem 2.3, we know that

$$
\frac{d}{d t}\left(t^{-\frac{n}{2}} e^{l_{+}\left(\gamma_{V}(t), t\right)} \mathcal{L}_{+} J_{V}(t)\right) \leq 0
$$

for $V \in \Omega(t)$. It follows that

$$
\frac{d}{d t}\left(t^{-\frac{n}{2}} e^{l_{+}\left(\gamma_{V}(t), t\right)} \mathcal{L}_{+} J_{V}(t) e^{-2|V|_{g(0)}^{2}}\right) \leq 0
$$

for $V \in \Omega(t)$. Moreover, since we have $\Omega\left(t_{2}\right) \subset \Omega\left(t_{1}\right)$,

$$
\widetilde{\mathcal{V}}_{+}\left(t_{2}\right) \leq \widetilde{\mathcal{V}}_{+}\left(t_{1}\right)
$$

for $t_{1}<t_{2}$. We calculate that

$$
\begin{aligned}
\lim _{t \rightarrow 0+} l_{+}\left(\gamma_{V}(t), t\right) & =\lim _{t \rightarrow 0+} \frac{1}{2 \sqrt{t}} \int_{0}^{t} \sqrt{\eta}\left(R\left(\gamma_{V}(\eta), \eta\right)+\left|\frac{d \gamma_{V}}{d \eta}\right|^{2}\right) d \eta \\
& =\lim _{t \rightarrow 0+} t\left(R\left(\gamma_{V}(t), t\right)+\left|\frac{d \gamma_{V}}{d t}\right|^{2}\right) \\
& =|V|_{g(0)}^{2} .
\end{aligned}
$$

Let $J_{i}^{V}(t), i=1, \cdots, n$, be $\mathcal{L}_{+}$-Jacobi fields along $\gamma_{V}(t)$ with

$$
J_{i}^{V}(0)=0,\left(\nabla_{V} J_{i}^{V}\right)(0)=E_{i}^{0},
$$


where $\left\{E_{i}^{0}\right\}_{i=1}^{n}$ is an orthonormal basis for $T_{x} M$ with respect to $g(0)$. Since $\left(\nabla_{V} J_{i}^{V}\right)(0)=E_{i}^{0}$ and $V=\lim _{t \rightarrow 0} \sqrt{t} \gamma_{V}^{\prime}(t)$, we get

$$
\lim _{t \rightarrow 0^{+}} \frac{\mathcal{L}_{+} J_{V}(t)}{t^{\frac{n}{2}}}=\lim _{t \rightarrow 0^{+}} \frac{\sqrt{\operatorname{det}\left(\left\langle 2 \sqrt{t} E_{i}(t), 2 \sqrt{t} E_{j}(t)\right\rangle_{g(0)}\right)}}{t^{\frac{n}{2}}}=2^{n},
$$

and we conclude that

$$
\lim _{t \rightarrow 0^{+}} t^{-\frac{n}{2}} e^{l_{+}\left(\gamma_{V}(t), t\right)} \mathcal{L}_{+} J_{V}(t)=2^{n} e^{|V|_{g(0)}^{2}} .
$$

Hence

$$
\lim _{t \rightarrow 0^{+}} \widetilde{\mathcal{V}}_{+}(t) \leq \int_{T_{p} M} 2^{n} e^{-|V|_{g(0)}^{2}} d x(V)=(4 \pi)^{\frac{n}{2}}
$$

If $\widetilde{\mathcal{V}}_{+}\left(t_{1}\right)=\widetilde{\mathcal{V}}_{+}\left(t_{2}\right)$ for any $0<t_{1}<t_{2}$, then $d \mathcal{V}_{+}\left(t_{1}\right)=d \mathcal{V}_{+}\left(t_{2}\right)$ for any $0<t_{1}<t_{2}$ So $\left(M^{n}, g(t)\right)$ must be a gradient expanding soliton by Theorem 2.3 and Theorem 3 in [4]; i.e. we have

$$
R c+H e s s\left(-l_{+}\right)=-\frac{g}{2 t}
$$

for some smooth function $l_{+}$on $M^{n}$. Let $\phi_{t}: M \rightarrow M, 0<t \leq \bar{t}$ be the oneparameter family of diffeomorphisms obtained by

$$
\frac{d \phi_{t}}{d t}=\nabla l_{+} \quad \text { and } \quad \phi_{\bar{t}}=I d
$$

We consider $h(t)=\frac{\bar{t}}{t} \phi_{t}^{*} g(t)$ and calculate

$$
\begin{aligned}
\frac{d h}{d t} & =-\frac{\bar{t}}{t^{2}} \phi_{t}^{*} g(t)+\frac{\bar{t}}{t} \phi_{t}^{*} \mathcal{L}_{\frac{d \phi_{t}}{d t}}(g(t))-2 \frac{\bar{t}}{t} \phi_{t}^{*} R c(g(t)) \\
& =-\frac{\bar{t}}{t^{2}} \phi_{t}^{*} g(t)+\frac{\bar{t}}{t} 2 H \operatorname{ess}\left(l_{+}\right)+\frac{\bar{t}}{t} \phi_{t}^{*}\left(\frac{g}{t}-2 \operatorname{Hess}\left(l_{+}\right)\right)=0 .
\end{aligned}
$$

It follows that

$$
g(t)=\frac{t}{\bar{t}}\left(\phi_{t}^{-1}\right)^{*} g(\bar{t}) .
$$

Suppose that there is some $(y, \bar{t})$ with $|R m|(y, \bar{t})=K>0$. We have $|R m|\left(\phi_{t}^{-1}(y), t\right)$ $=\frac{K \bar{t}}{t}$, and these curvatures are not bounded as $t \rightarrow 0$, which is a contradiction. Then we have

$$
\operatorname{Hess}\left(l_{+}\right)=\frac{1}{2 t} g .
$$

Thus $l_{+}$is a strictly convex function. Similar arguments to Lemma 2.3 in [14] can show that

$$
l_{+}(y, t) \geq e^{-2 c t} \frac{d_{g(0)}(x, y)}{4 t}-\frac{n c}{3} t
$$

if $R c \geq-c g$ on $[0, t]$, so that $l_{+}(y, t)$ have only a minimum point in $M^{n}$. Hence $M^{n}$ is diffeomorphic to $\mathbb{R}^{n}$.

Since $\widetilde{\mathcal{V}}_{+}(t)$ is monotone nonincreasing, $\widetilde{\mathcal{V}}_{+}(t)$ is independent of $t$ if $\widetilde{\mathcal{V}}_{+}(\bar{t})=$ $(4 \pi)^{\frac{n}{2}}$ for some time $\bar{t}>0$. Then we derive that $\left(M^{n}, g(\bar{t})\right)$ is isometric to $\mathbb{R}^{n}$. By Theorem 1 in [4], the Ricci flow $\left(M^{n}, g(t)\right)_{t \in[0, T]}$ is in fact isometric to flat Euclidean space $\mathbb{R}^{n}$ for all $t \in[0, T]$.

Finally, we give the proof of Theorem 1.2 . 
Proof of Theorem 1.2. We denote $\gamma_{v}^{j}(t)$ to be the minimal $\mathcal{L}_{+}$-geodesic with respect to $g_{j}(t)$ which starts from $\left(x_{j}, 0\right)$ and satisfies $\lim _{t \rightarrow 0} \sqrt{t} \frac{d \gamma_{V}^{j}(t)}{d t}=V$.

We have $\gamma_{\sqrt{\lambda_{j}^{-1}} V}^{j}(t)=\gamma_{V}\left(\lambda_{j}^{-1} t\right), l_{+}^{j}(y, t)=l_{+}\left(y, \lambda_{j}^{-1} t\right)$, and $\mathcal{L}_{+} J_{V}^{j}(t) d x_{g_{j}(0)}(V)$ $=\left(\lambda_{j}^{-1}\right)^{-\frac{n}{2}} \mathcal{L}_{+} J_{\sqrt{\lambda_{j}} V}\left(\lambda_{j}^{-1} t\right) d x_{g(0)}\left(\sqrt{\lambda_{j}} V\right)$. Hence

$$
\begin{aligned}
\tilde{\mathcal{V}}_{+}^{j}(t)= & \int_{T_{x} M^{n}}(t)^{-\frac{n}{2}} e^{l_{+}^{j}\left(\gamma_{V}(t), t\right)} \mathcal{L}_{+} J_{V}^{j}(t) e^{-2|V|_{g_{j}(0)}^{2}} d x_{g_{j}(0)}(V) \\
= & \int_{T_{x} M^{n}}\left(\lambda_{j}^{-1} t\right)^{-\frac{n}{2}} e^{l_{+}\left(\gamma_{\sqrt{\lambda_{j}} V}\left(\lambda_{j}^{-1} t\right), \lambda_{j}^{-1} t\right)} \\
& \quad \times \mathcal{L}_{+} J \sqrt{\lambda_{j} V}\left(\lambda_{j}^{-1} t\right) e^{-2\left|\sqrt{\lambda_{j}} V\right|_{g(0)}^{2}} d x_{g(0)}\left(\sqrt{\lambda_{j}} V\right) \\
= & \widetilde{\mathcal{V}}_{+}\left(\lambda_{j}^{-1} t\right) .
\end{aligned}
$$

Note that $\mathcal{L}_{+}^{j} \exp ^{-1}(y, t)=\sqrt{\lambda_{j}^{-1}} \mathcal{L}_{+} \exp ^{-1}\left(y, \lambda_{j}^{-1} t\right)$. We can also prove Theorem 1.2 by using the form (1.9)

$$
\begin{aligned}
\tilde{\mathcal{V}}_{+}^{j}(t) & =\int_{M} t^{-\frac{n}{2}} e^{l_{+}^{j}(y, t)} e^{-2\left|\mathcal{L}_{+}^{j} \exp ^{-1}(y, t)\right|_{g_{j}(0)}^{2}} d v o l_{g_{j}(t)}(y) \\
& =\int_{M}\left(\lambda_{j}^{-1} t\right)^{-\frac{n}{2}} e^{l_{+}\left(y, \lambda_{j}^{-1} t\right)} e^{-2\left|\mathcal{L}_{+} \exp ^{-1}\left(y, \lambda_{j}^{-1} t\right)\right|_{g(0)}^{2}} d v o l_{g\left(\lambda_{j}^{-1} t\right)}(y) \\
& =\widetilde{\mathcal{V}}_{+}\left(\lambda_{j}^{-1} t\right) .
\end{aligned}
$$

\section{Appendix}

In this section, we give the proofs of Theorem 2.1 and Theorem 2.2, which are proved in 3 , for the sake of completeness.

Proof of Theorem 2.1 and Theorem 2.2. Note that (2.1) and (2.4) differ from the first and second variation of Perelman's $\mathcal{L}$-length [11 only by the sign in front of $R c$, since Perelman's $\mathcal{L}$-length is defined under the backward Ricci flow (1.1). The calculations are almost the same as [11, and we omit the details here.

If $\gamma(t)$ is the mimimal $\mathcal{L}_{+}$-geodesic, it follows from (2.1) that

$$
\nabla L_{+}=2 \sqrt{t} X
$$

Now using the geodesic equation, we have

$$
\begin{aligned}
& \frac{d}{d t}\left(R(\gamma(t), t)+|X(t)|^{2}\right) \\
= & \frac{\partial}{\partial t} R+\nabla R \cdot X-2 R c(X, X)+2\left\langle\nabla_{X} X, X\right\rangle \\
= & \frac{\partial}{\partial t} R+2 \nabla R \cdot X+2 R c(X, X)-\frac{1}{t}|X|^{2} .
\end{aligned}
$$

Hence

$$
\frac{d}{d t}\left(R(\gamma(t), t)+|X(t)|^{2}\right)=H(x)-\frac{1}{t}\left(R+|X|^{2}\right),
$$


where $H(X)=\frac{\partial R}{\partial t}+2 \nabla R \cdot X+2 R c(X, X)+\frac{R}{t}$, and this gives (2.3) immediately. Let $\widetilde{Y}$ be a vector field along $\gamma$ that satisfies the ODE

$$
\left\{\begin{array}{l}
\nabla_{X} \tilde{Y}(\eta)=R c(\tilde{Y}(\eta), \cdot)+\frac{1}{2 \eta} \tilde{Y}(\eta), \eta \in[0, t], \\
\tilde{Y}(0)=Y(0)=0 .
\end{array}\right.
$$

Then we have $|\widetilde{Y}(\eta)|^{2}=\frac{\eta}{t}|\widetilde{Y}(t)|^{2}$. It follows that

$H \operatorname{Hess} L_{+}(\tilde{Y}, \tilde{Y}) \leq \frac{1}{\sqrt{t}}|\tilde{Y}(t)|^{2}+\int_{0}^{t} \sqrt{\eta}\left(\operatorname{HessR}(\tilde{Y}, \tilde{Y})-2 R(X, \tilde{Y}, X, \tilde{Y})+2|R c(\cdot, \tilde{Y})|^{2}\right.$

$$
\left.\left.+\frac{2}{\eta} R c(\widetilde{Y}, Y)+\left(4 \nabla_{\widetilde{Y}} R c(\widetilde{Y}, X)-2 \nabla_{X} R c(\widetilde{Y}, \widetilde{Y})\right)\right)+2 \nabla_{X} R c(\widetilde{Y}, \widetilde{Y})\right) d \eta
$$

Since

$$
\frac{d}{d t}(R c(\widetilde{Y}, \tilde{Y}))=\frac{\partial R c}{\partial t}(\widetilde{Y}, \widetilde{Y})+\nabla_{X} R c(\widetilde{Y}, \widetilde{Y})+2|R c(\cdot, \tilde{Y})|^{2}+\frac{1}{t} R c(\tilde{Y}, \tilde{Y}),
$$

we get

$$
\begin{aligned}
& 2 \int_{0}^{t} \sqrt{\eta} \nabla_{X} R c(\tilde{Y}, \tilde{Y}) \\
= & 2 \sqrt{t} R c(\widetilde{Y}, \tilde{Y})-\int_{0}^{t} \sqrt{\eta}\left(3 \frac{R c(\tilde{Y}, \tilde{Y})}{\eta}+2 \frac{\partial R c}{\partial t}(\tilde{Y}, \tilde{Y})+4|R c(\cdot, \tilde{Y})|^{2}\right) d \eta .
\end{aligned}
$$

Substituting (3.4) into (3.3), we have

$$
H e s s L_{+}(\widetilde{Y}, \widetilde{Y}) \leq \frac{|\widetilde{Y}|^{2}}{\sqrt{t}}+2 \sqrt{t} R c(\widetilde{Y}, \widetilde{Y})-\int_{0}^{t} \sqrt{\eta} H(X, \widetilde{Y}) d \eta
$$

where $H(X, \tilde{Y})=-H \operatorname{ess} R(\tilde{Y}, \tilde{Y})+2 R(X, \tilde{Y}, X, \tilde{Y})+2|R c(\widetilde{Y}, \cdot)|^{2}+\frac{R c(\widetilde{Y}, \tilde{Y})}{t}+$ $2 \frac{\partial R c}{\partial t}(\tilde{Y}, \tilde{Y})-4 \nabla_{\tilde{Y}} R c(\tilde{Y}, X)+4 \nabla_{X} R c(\tilde{Y}, \tilde{Y})$. The equality holds in (2.6) if and only if the vector field $\widetilde{Y}$ satisfying (2.16) is an $\mathcal{L}_{+}$-Jacobi field. Tracing (2.6) in $Y$ yields

$$
\Delta L_{+} \leq \frac{n}{\sqrt{t}}+2 \sqrt{t} R-\frac{1}{t} K
$$

By (2.2), we have

$$
\left|\nabla L_{+}\right|^{2}=-4 t R+4 t\left(R+|X|^{2}\right)
$$

and

$$
\begin{aligned}
\frac{\partial L_{+}}{\partial t}(y, t) & =\frac{d L_{+}(\gamma(t), t)}{d t}-\nabla L_{+} \cdot X \\
& =\sqrt{t}\left(R+|X|^{2}\right)-2 \sqrt{t}|X|^{2} \\
& =2 \sqrt{t} R-\sqrt{t}\left(R+|X|^{2}\right) .
\end{aligned}
$$

Substituting (2.3) into (3.7) and (3.8), we have

$$
\left|\nabla L_{+}\right|^{2}=-4 t R+\frac{2}{\sqrt{t}} L_{+}+\frac{4}{\sqrt{t}} K
$$

and

$$
\frac{\partial L_{+}}{\partial t}=2 \sqrt{t} R-\frac{1}{t} K-\frac{1}{2 t} L_{+}
$$


Then (2.7)-(2.11) follow from (3.6), (3.9) and (3.10).

\section{REFERENCES}

1. B. Chow, S. C. Chu, D. Glickenstein, C. Guenther, J. Isenberg, T. Ivey, D. Knopf, P. Lu, F. Luo, L. Ni, The Ricci flow: Techniques and applications. Part I, Mathematical Surveys and Monographs, vol. 135, American Mathematical Society, Providence, RI, 2007, Geometric aspects. MR2302600 (2008f:53088)

2. J. Enders, R. Müller, P. M. Topping, On Type I singularities in Ricci flow, Comm. Anal. Geom. 19 (2011), no. 5, 905-922. MR2886712

3. M. Feldman, T. Ilmanen, L. Ni, Entropy and reduced distance for Ricci expanders, J. Geom. Anal. 15 (2005), 49-62. MR2132265 (2006b:53091)

4. Kotschwar, Brett L. Backwards uniqueness for the Ricci flow. Int. Math. Res. Not. IMRN (2010), no. 21, 4064-4097. MR2738351 (2012c:53100)

5. J. Lott, On the long time behavior of type III Ricci flow solutions, Math. Ann. 339 (2007), 627-666. MR2336062(2008i:53093)

6. L. Ma, Ricci expanders and Type III Ricci flow, http://arxiv.org/abs/1008.0711v1.

7. L. Ma, A complete proof of Hamilton's conjecture, http://arxiv.org/abs/1008.1576v1.

8. J. Morgan, G. Tian, Ricci flow and the Poincaré conjecture, Clay Mathematics Monographs, Volume 3, American Mathematical Society, Providence, RI, 2007. MR2334563 (2008d:57020)

9. R. Hamilton, The formation of singularities in the Ricci flow, Surveys in differential geometry, Vol. II (Cambridge, MA, 1993), Internat. Press, Cambridge, MA, 1995. 7-136. MR.1375255 (97e:53075)

10. R. Hamilton, Three-manifolds with positive Ricci curvature. J. Differential Geom. 17 (1982), no. 2, 255-306. MR664497(84a:53050)

11. G. Perelman, The entropy formula for the Ricci flow and its geometric applications. http://arxiv.org/abs/math/0211159.

12. G. Perelman, Ricci flow with surgery on three-manifolds. http://arxiv.org/abs/ /math/0303109v1.

13. G. Perelman, Finite time extinction for the solutions to the Ricci flow on certain threemanifold. http://arxiv.org/abs/math/0307245.

14. R. Ye, On the l-function and the reduced volume of Perelman. I, Trans. Amer. Math. Soc. 360 (2008), no. 1, 507-531. MR2342013(2008j:53056)

School of Mathematics and Statistics, Huazhong Normal University, Wuhan, 430079, People's Republic of China

E-mail address: math.chengliang@gmail.com

School of Mathematics and Statistics, Wuhan University, Wuhan, 430072, People's RePublic of China

E-mail address: anqiangzhu@yahoo.com.cn 